\title{
Evaluation of a 1-h 75-g oral glucose tolerance test in the diagnosis of gestational diabetes
}

\author{
M.A.A. Campos ${ }^{1,2}$, A.A.J. Reichelt ${ }^{3}$, C. Façanha ${ }^{4}$, A.C. Forti ${ }^{4,5}$ and M.I. Schmidt ${ }^{1}$ \\ 1Programa de Pós-Graduação em Epidemiologia, Faculdade de Medicina, Universidade Federal do Rio \\ Grande do Sul, Porto Alegre, RS, Brasil \\ ${ }^{2}$ Hospital Nossa Senhora da Conceição, Porto Alegre, RS, Brasil \\ ${ }^{3}$ Hospital de Clínicas de Porto Alegre, Porto Alegre, RS, Brasil \\ ${ }^{4}$ Centro de Diabetes e Hipertensão, Secretaria da Saúde do Estado do Ceará, Fortaleza, CE, Brasil \\ ${ }^{5}$ Departamento de Clínica Médica, Faculdade de Medicina, Universidade Federal do Ceará, Fortaleza, \\ CE, Brasil
}

Correspondence to: M.A.A. Campos, Rua Carlos de Carvalho, 82, 90630-040 Porto Alegre, RS, Brasil

Fax: +55-51-3330-7048. E-mail: ameliaac@terra.com.br

\begin{abstract}
In order to evaluate the performance of a 1-h 75-g oral glucose tolerance test (OGTT) for the diagnosis of gestational diabetes mellitus (GDM), a cohort of 4998 women, 20 years or older, without previous diabetes being treated in prenatal care clinics in Brazil answered a questionnaire and performed a 75-g OGTT including fasting, 1-h and 2-h glucose measurements between their 24th and 28th gestational weeks. Pregnancy outcomes were transcribed from medical registries. GDM was defined according to WHO criteria (fasting: $\geq 126 \mathrm{mg} / \mathrm{dL} ; 2-\mathrm{h}$ value: $\geq 140 \mathrm{mg} / \mathrm{dL}$ ) and macrosomia as a birth weight equal to or higher than $4000 \mathrm{~g}$. Areas under the receiver operator characteristic curve (AUC) were compared and diagnostic properties of various cutoff points were evaluated. The AUCs for the prediction of macrosomia were $0.606(0.572-0.637)$ for the $1-\mathrm{h}$ and 0.589 (0.557$0.622)$ for the 2-h plasma glucose test. Similar predictability was demonstrable regarding combined adverse outcomes: 0.582 (0.559-0.604) for the 1-h test and $0.572(0.549-0.595)$ for the 2-h test. When the 1-h glucose test was evaluated against a diagnosis of GDM defined by the 2-h glucose test, the AUC was 0.903 (0.886-0.919). The cut-off point that maximized sensitivity $(83 \%)$ and specificity (83\%) was $141 \mathrm{mg} / \mathrm{dL}$, identifying $21 \%$ of the women as positive. A cut-off point of $160 \mathrm{mg} / \mathrm{dL}$, with lower sensitivity (62\%), had higher specificity (94\%), labeling $8.6 \%$ as positive. Detection of GDM can be done with a 1-h 75-g OGTT: the value of $160 \mathrm{mg} / \mathrm{dL}$ has the same diagnostic performance as the conventional 2-h value (140 $\mathrm{mg} / \mathrm{dL}$ ). The simplification of the test may improve coverage and timing of the diagnosis of GDM.
\end{abstract}

Key words: Gestational diabetes; Diagnosis; Macrosomia; 1-h OGTT; Sensitivity; Specificity

Research supported in part by the Brazilian Ministry of Health, the Pan-American Health Organization (PAHO), FAPERGS, CNPq, PRONEX (Award for Groups of Excellence), Bristol-Myers Squibb Foundation, and FIPE-HCPA (Fund for the Support of Research of the Hospital de Clínicas de Porto Alegre).

Received September 20, 2007. Accepted June 16, 2008

\section{Introduction}

There is a controversy concerning the diagnosis of gestational diabetes mellitus (GDM), defined as glucose intolerance first recognized in pregnancy (1). The two most common criteria adopted are the one proposed by the World Health Organization (WHO) (1), based on the 2-h 75-g oral glucose tolerance test (OGTT), and another recommended by the American Diabetes Association $(2,3)$, based on the 3-h 100-g OGTT. Evaluation of these criteria has been done in different populations and settings, but recognition of the continuum risk of adverse events related to post-load glucose values has limited attempts to reach consensual criteria (4-6).

Nevertheless, validity of detecting and effectively treating GDM has gained support by results from a recently concluded clinical trial demonstrating that treatment of GDM, as defined by WHO criteria, was associated with 
reduced risk of adverse pregnancy outcomes (7).

The objective of the present study was to evaluate a shorter version of the 2-h 75-g OGTT, a 1-h 75-g test, for the diagnosis of gestational diabetes by assessing its capacity to predict adverse maternal and fetal outcomes.

\section{Material and Methods}

A cohort study of consecutive pregnant women was conducted in general prenatal care units of six state capitals in Brazil from 1991 to 1995. A total of 5564 women aged 20 years or more with no diagnosis of previous diabetes and who were at about 21 to 28 weeks of pregnancy agreed to participate. Local institutional Ethics Committees approved the study protocol. Due to the low risk of blood sampling collection, written informed consent was not required by the Ethics Committees at that time.

At enrollment, we applied a standardized questionnaire and a protocol with anthropometric measurements made in duplicate. Body mass index was calculated as weight $(\mathrm{kg})$ divided by the square of height $\left(\mathrm{m}^{2}\right)$. The interviewer defined ethnicity according to the color of the participant's skin as white, African Brazilian, mixed (an admixture of African Brazilian and white) or other.

Table 1. Characteristics of the 4998 pregnant women who participated in the Brazilian Study of Gestational Diabetes, 19911995.

\begin{tabular}{lc}
\hline Chararacteristic & $\mathrm{N}(\%)$ \\
\hline Age & \\
$20-24$ years & $1650(33 \%)$ \\
$25-29$ years & $1585(32 \%)$ \\
$\geq 30$ years & $1763(35 \%)$ \\
Ethnicity & \\
White & $2442(44 \%)$ \\
Mixed & $2339(42 \%)$ \\
African Brazilian & $755(13.6 \%)$ \\
Other & $25(0.4 \%)$ \\
Pre-pregnancy BMI (kg/m $\left.{ }^{2}\right)$ & \\
$<18.5$ & $276(6 \%)$ \\
$18.5-24.9$ & $3160(66 \%)$ \\
$25-29.9$ & $1031(21 \%)$ \\
$\geq 30$ & $316(7 \%)$ \\
Education & \\
$<8$ years & \\
$8-11$ years & $2190(44 \%)$ \\
$>11$ years & $2306(46 \%)$ \\
Smoked during pregnancy & $490(10 \%)$ \\
Short stature $(<1.5 \mathrm{~m})$ & $867(17 \%)$ \\
\hline
\end{tabular}

Data are reported as number with percent in parentheses. $\mathrm{BMI}=$ body mass index.
A modified 2-h 75-g OGTT, which included a 1-h plasma glucose measurement based mainly on the WHO panel technical recommendations (1), was performed between the 24th and 28th weeks of pregnancy. Fasting, 1-h and 2-h plasma glucose concentrations were measured by an enzymatic method (8).

GDM was defined according to the WHO panel recommendations: fasting plasma glucose equal to or exceeding $126 \mathrm{mg} / \mathrm{dL}$ or 2-h plasma glucose equal to or exceeding $140 \mathrm{mg} / \mathrm{dL}$ during the 75-g OGTT (1).

We followed participants until delivery and obtained outcome data from medical registries. Macrosomia was defined as a birth weight equal to or higher than $4000 \mathrm{~g}$. Hypertension, preeclampsia or eclampsia were ascertained by chart review and classified according to the National High Blood Pressure Education Program Working Group (9). A composite of adverse outcomes was defined as the presence of macrosomia, preeclampsia or perinatal death.

A total of 4998 women completed the OGTT test. Birth weight was available for 4356 newborns; data for the composite of pregnancy outcomes were available for 3941 women. Sensitivity, specificity and the percentage of women categorized as positive for each value of the 1-h glucose test were calculated for various outcomes (GDM, macrosomia and the composite of outcomes) (10).

We performed analyses, including the $95 \%$ confidence interval $(95 \% \mathrm{Cl})$ for proportions, using version 10 SPSS statistical package. We plotted receiver operator characteristic (ROC) curves, estimated the area under the curve (AUC) and compared curves using the Analyse-it for Microsoft Excel (www.analyse-it.com).

\section{Results}

The baseline data for the 4998 women who completed the OGTT are presented in Table 1. It can be seen that $65 \%$ of the sample were 20 to 30 years old, only $35 \%$ being 30 years or older; $44 \%$ were white, $42 \%$ of an admixture of African Brazilian and white, and $14 \%$ African Brazilian or other ethnic group. Most had normal pre-pregnancy body mass index (66\%), $28 \%$ were overweight or obese and $6 \%$ were underweight. Almost half of the sample went to school for less than 8 years, $17 \%$ were smoking at the current pregnancy and $15 \%$ had a stature $<1.5 \mathrm{~m}$.

In order to evaluate the overall performance of the 1-h versus the 2-h plasma glucose determinations following a 75-g glucose load, we compared the AUC of the ROC curves for the prediction of adverse pregnancy outcomes. Figure $1 \mathrm{~A}$ shows that, for fetal macrosomia, the 1-h and 2h curves virtually overlapped: the AUC was $0.606(95 \% \mathrm{Cl}$ $=0.572-0.637)$ for the $1-\mathrm{h}$ and $0.589(95 \% \mathrm{Cl}=0.557-$ 
$0.622)$ for the 2 -h glucose test $(P>0.05)$. Figure $1 \mathrm{~B}$ shows similar results for a composite of pregnancy outcomes: the AUC for the $1-\mathrm{h}$ value was $0.582(95 \% \mathrm{Cl}=0.559-0.604)$ and for the $2-\mathrm{h}$ value, $0.572(95 \% \mathrm{Cl}=0.549-0.595 ; \mathrm{P}>$ 0.05).

We next examined the performance of the 1-h test against a diagnosis of GDM defined by the conventional 2$\mathrm{h}$ test. Figure 2 shows very high predictability: $0.903(95 \% \mathrm{Cl}$ $=0.886-0.919)$. As indicated by the diagonal line that begins at $100 \%$ sensitivity and goes to $100 \% 1$-specificity, the cut-off point that jointly maximized sensitivity (83\%) and specificity (83\%) was $141 \mathrm{mg} / \mathrm{dL}$.

Table 2 contains the sensitivity and specificity cut-off points for the 1-h post-load glucose for the diagnosis of
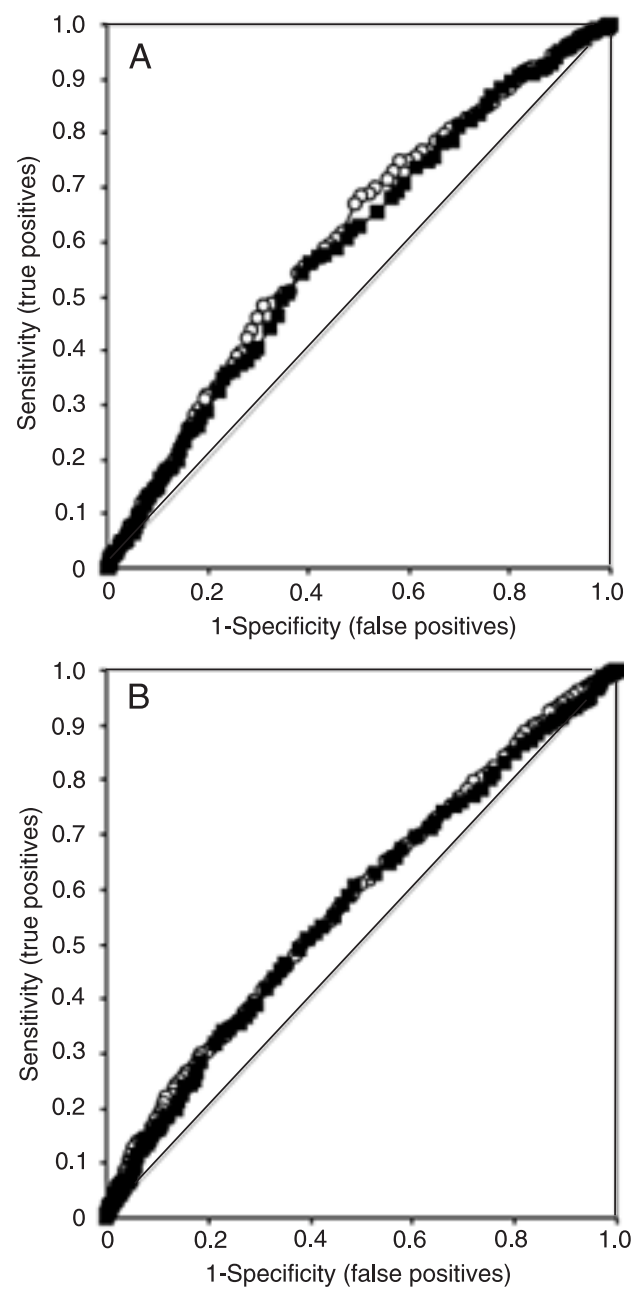

Figure 1. Receiver operator characteristic curves comparing performance of 1-h and 2-h oral glucose tolerance test for the prediction of macrosomia $(A)$ and for the prediction of combined adverse outcomes (B). Open symbols = 1-h plasma glucose concentration; closed symbols $=2$-h plasma glucose concentration.
GDM and macrosomia. Diagnostic properties for the conventional 2-h glucose test are reported for comparison.

The value of $140 \mathrm{mg} / \mathrm{dL}$, rounded from $141 \mathrm{mg} / \mathrm{dL}$ had a sensitivity of $85 \%$ and a specificity of $80 \%$ for the detection of GDM; this cut-off point detected more macrosomic fetuses than the conventional 2-h diagnostic cut-off point (36 vs 13\%). However, if this cut-off point were used instead of the 2-h value, many more women would be labeled as GDM (22 vs 7.6\%).

The value of $180 \mathrm{mg} / \mathrm{dL}$ reached very high specificity in detecting GDM (99\%) and macrosomia (97\%), but the trade-off was low sensitivities (31 and $5 \%$, respectively). This high cut-off point would identify only $3.2 \%$ of the women as GDM.

An intermediate value $(160 \mathrm{mg} / \mathrm{dL})$ had high specificity $(94 \%)$ and reasonable sensitivity (62\%) for the detection of GDM, labeling fewer women (8.6\%) as having GDM. This cut-off point had similar sensitivity to detect macrosomia as the 2-h test (14 vs $13 \%$ ), with similar high specificity (90 vs $93 \%$ ).

Because the ability of fasting plasma glucose to detect macrosomia and a composite of pregnancy outcomes was similar to that found for the 1-h glucose test (data not shown), we also evaluated the performance of fasting plasma glucose for detecting GDM. The AUC was 0.768 $(95 \% \mathrm{Cl}=0.743-0.792)$, which is lower than that reported above for the 1-h glucose test $(P<0.001)$. In fact, combining different fasting values with the 1-h cut-off point of 160 $\mathrm{mg} / \mathrm{dL}$ did not improve its diagnostic performance. For

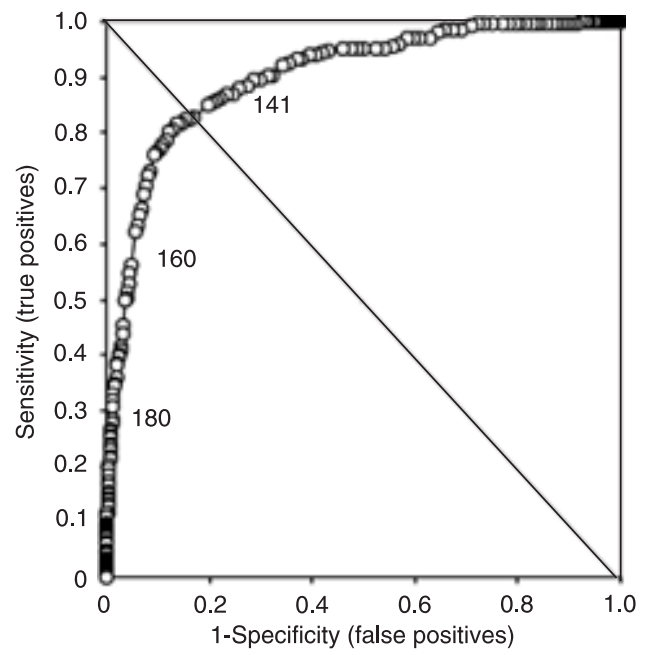

Figure 2. Receiver operator characteristic curve assessing performance of 1-h oral glucose tolerance test for the detection of gestational diabetes. The diagonal line denotes the point that maximizes sensitivity and specificity. 
Table 2. Performance of 1-h plasma glucose test for the prediction of gestational diabetes and macrosomia.

\begin{tabular}{|c|c|c|c|c|c|}
\hline \multirow{2}{*}{$\begin{array}{l}\text { OGTT } \\
\text { cut-off point } \\
(\mathrm{mg} / \mathrm{dL})\end{array}$} & \multirow[t]{2}{*}{ Positive test } & \multicolumn{2}{|c|}{ Gestational diabetes $(2-\mathrm{h} \geq 140 \mathrm{mg} / \mathrm{dL})$} & \multicolumn{2}{|c|}{ Macrosomia (birth weight $\geq 4 \mathrm{~kg}$ ) } \\
\hline & & Sensitivity $(95 \% \mathrm{Cl})$ & Specificity $(95 \% \mathrm{Cl})$ & Sensitivity $(95 \% \mathrm{Cl})$ & Specificity $(95 \% \mathrm{Cl})$ \\
\hline \multicolumn{6}{|l|}{$1-h$} \\
\hline 120 & $47 \%$ & $95 \%(92-97)$ & $54 \%(38-40)$ & $67 \%(61-72)$ & $51 \%(49-52)$ \\
\hline 130 & $34 \%$ & $89 \%(86-92)$ & $69 \%(67-70)$ & $50 \%(45-56)$ & $65 \%(63-66)$ \\
\hline 140 & $22 \%$ & $85 \%(81-88)$ & $80 \%(79-81)$ & $36 \%(31-41)$ & $76 \%(74-77)$ \\
\hline 150 & $15 \%$ & $77 \%(73-81)$ & $90 \%(89-90)$ & $23 \%(18-28)$ & $85 \%(84-86)$ \\
\hline 160 & $8.6 \%$ & $62 \%(57-67)$ & $94 \%(94-95)$ & $14 \%(11-19)$ & $90 \%(89-91)$ \\
\hline 170 & $5.4 \%$ & $41 \%(36-46)$ & $97 \%(97-98)$ & $8.0 \%(5-12)$ & $95 \%(94-95)$ \\
\hline 180 & $3.2 \%$ & $31 \%(26-35)$ & $99 \%(98-99)$ & $5.4 \%(3-8)$ & $97 \%(96-97)$ \\
\hline \multicolumn{6}{|l|}{ 2-h } \\
\hline 140 & $7.6 \%$ & & & $13 \%(10-17)$ & $93 \%(91-93)$ \\
\hline
\end{tabular}

OGTT = oral glucose tolerance test.

instance, combining the 1 -h cut-off point of $160 \mathrm{mg} / \mathrm{dL}$ with a fasting value of $80 \mathrm{mg} / \mathrm{dL}$, which in isolation had similar frequency of positive testing as the 1 -h value of $160 \mathrm{mg} / \mathrm{dL}$ (8.6 vs $8.5 \%)$, produced a sensitivity of $55 \%(95 \% \mathrm{Cl}=50$ $60 \%)$ and a specificity of $86 \%(95 \% \mathrm{Cl}=85-87 \%)$. For the detection of macrosomia, this combination provided a sensitivity of $15 \%(95 \% \mathrm{Cl}=12-19 \%)$ and a specificity of $92 \%$ $(95 \% \mathrm{Cl}=91-93 \%)$.

\section{Discussion}

The results of this study based on data for a Brazilian cohort of 4998 pregnant women show that the 1-h 75-g OGTT has similar properties for the diagnosis of GDM as the classic 2-h 75-g OGTT.

The 1-h test that maximized both sensitivity and specificity for the detection of GDM (141 mg/dL) approaches that originally described by O'Sullivan et al. (11) for the 50 $\mathrm{g} 1$-h test (130 mg/dL for blood glucose) and which is still largely used for the screening of GDM. Properties for the $141 \mathrm{mg} / \mathrm{dL} 1$-h test (sensitivity and specificity of 83\%) are consistent with those usually required for screening of GDM, as well as is the proportion of women classified as positive (22\%), i.e., women who would require the diagnostic 2-h test.

For diagnostic purposes, however, it is desirable to have high specificity, which requires higher cut-off points. The cut-off point of $180 \mathrm{mg} / \mathrm{dL}$ reached a specificity of almost $100 \%$ but, consequently, very low sensitivity (31\%). It coincides with the 1-h cut-off point proposed by Carpenter and Coustan for the diagnosis of GDM (2). Similarly, high specificity (96\%) and low sensitivity (36\%) were also reported by Landy et al. (12) for a 1-h 50-g cut-off point of $186 \mathrm{mg} / \mathrm{dL}$, proposing it as a diagnostic test for GDM.

We also found reasonably high specificity $(94 \%)$ for the cut-off point of $160 \mathrm{mg} / \mathrm{dL}$, suggesting its potential use for the diagnosis of GDM. The sensitivity of this cut-off point was $62 \%$ and the percent labeled as positive, $8.6 \%$, just a little higher than that obtained with the 2-h glucose criteria (7.6\%) (13). The specificity for the detection of macrosomia $(90 \%)$ was similar to that reported by Mello et al. (14) (99\%) for the detection of abnormal neonatal anthropometric measures.

Our finding of similar AUC for the 1-h and the 2-h glucose test in the prediction of macrosomia and combined adverse outcomes supports the view that the 1-h glucose test could replace the 2-h glucose test for the diagnosis of GDM. There are advantages of shortening the duration of the test. In addition to making it more convenient for the patient, it decreases associated costs of a longer test. As a result, it may allow greater screening coverage and GDM detection yield. Similar conclusions were also reached by Mello et al. (14), including the diagnostic properties supporting its use for the diagnosis of GDM with a cut-off point of $160 \mathrm{mg} / \mathrm{dL}$.

Maintaining the goal of keeping the patient for only $1 \mathrm{~h}$ in the laboratory for sample collection, but accepting increased costs and inconveniences of also taking a fasting sample, we investigated potential diagnostic gains of combining various cut-off points for fasting values. We found no combination that improved diagnostic properties.

Some limitations of our study deserve comment. The outcomes we chose are not specific for hyperglycemia and diagnostic properties for glucose values have barely satis- 
factory performances when evaluated against such outcomes. Another potential limitation is the lack of independence between the 1-h and the 2-h glucose values obtained during the same OGTT. However, our objective was to evaluate the need to remain in the laboratory for an extra hour in order to complete the conventional 2-h test. We also evaluated additional clinically relevant outcomes associated with GDM. Although some selection bias due to losses to follow-up could have been present, comparison of those lost to those included in the analyses did not indicate any major differences regarding risk factors for GDM or adverse pregnancy outcomes (5).

There are potential advantages of the 1-h 75-g OGTT for the diagnosis of GDM. When applied universally and adopting the $160 \mathrm{mg} / \mathrm{dL}$ as a diagnostic cut-off point, the yield of GDM cases is comparable to that generated by the WHO 2-h OGTT criteria (8.6 vs 7.6\%). Applied as a onestep diagnostic strategy, the 1-h test may allow earlier treatment for $8.6 \%$ of the women, and being simpler and less costly, may improve adherence to screening protocols. Furthermore, adopting a screening cut-off point of $140 \mathrm{mg} / \mathrm{dL}$ would permit reporting to $78 \%$ of the women that their screening test was negative. The remaining women (13\%), the "positive screenees", would then need a confirmatory test. If the $1-\mathrm{h}$ test result were available immediately, such women could complete the 2-h test at that moment or alternatively, a 2-h confirmatory test would need to be scheduled.

These advantages are potentially very relevant. Prompt diagnosis of GDM is an important goal, since it is a transient situation requiring intervention during a short period, rarely for more than 20 weeks.

\section{Acknowledgments}

The Brazilian Gestational Diabetes Study Group, Brazilian Ministry of Health: Romero Bezerra Barbosa, Laurenice P. Lima and Enrique Rivero Ortiz; Central Project Coordination: Maria Inês Schmidt, Angela J. Reichelt, Leandro Branchtein, Maria Cristina Gomes Matos, Sotero Mengue and Bruce B. Duncan; Quality Control Committee: Lúcia lochida, Laércio J. Franco and Ewaldo Russo; Study Centers: Porto Alegre: Angela J. Reichelt, Leandro Branchtein and Maria Cristina Gomes Matos; Salvador: Judith Maria Dias Pousada, Maria Margarida dos Santos Britto and Denise Barata; São Paulo: Tsuyoshi Yamashita and Cláudia Nogueira; Rio de Janeiro: Ethel R. Stambovsky Spichler and Cláudia Martins; Manaus: Margareth Mauro and Maria Cristina Tavares da Costa; Fortaleza: Adriana Costa e Forti and Eni Fleck de Paula Pessoa.

\section{References}

1. Alberti KG, Zimmet PZ. Definition, diagnosis and classification of diabetes mellitus and its complications. Part 1: diagnosis and classification of diabetes mellitus provisional report of a WHO consultation. Diabet Med 1998; 15: 539-553.

2. American Diabetes Association. Gestational diabetes mellitus. Diabetes Care 2007; 30 (Suppl 1): S7.

3. American College of Obstetricians and Gynecologists. Diabetes and pregnancy. Technical Bulletin No. 200; 1994.

4. Sacks DA, Greenspoon JS, Abu-Fadil S, Henry HM, WoldeTsadik G, Yao JF. Toward universal criteria for gestational diabetes: the 75-gram glucose tolerance test in pregnancy. Am J Obstet Gynecol 1995; 172: 607-614.

5. Schmidt MI, Duncan BB, Reichelt AJ, Branchtein L, Matos MC, Costa e Forti A, et al. Gestational diabetes mellitus diagnosed with a 2-h 75-g oral glucose tolerance test and adverse pregnancy outcomes. Diabetes Care 2001; 24: 1151-1155.

6. Sermer M, Naylor CD, Gare DJ, Kenshole AB, Ritchie JW, Farine $D$, et al. Impact of increasing carbohydrate intolerance on maternal-fetal outcomes in 3637 women without gestational diabetes. The Toronto Tri-Hospital Gestational Diabetes Project. Am J Obstet Gynecol 1995; 173: 146156.

7. Crowther CA, Hiller JE, Moss JR, McPhee AJ, Jeffries WS, Robinson JS. Effect of treatment of gestational diabetes mellitus on pregnancy outcomes. N Engl J Med 2005; 352:

\section{7-2486}

8. Trinder P. Determination of glucose in blood using glucose oxidase with an alternative glucose acceptor. Ann Clin Biochem 1969; 6: 24-27.

9. National High Blood Pressure Education Program Working Group. Report on high blood pressure in pregnancy. Am J Obstet Gynecol 1990; 163: 1691-1712.

10. Fletcher R, Fletcher S, Wagner EH. Epidemiologia clínica: elementos essenciais. Porto Alegre: Artes Médicas; 1996.

11. O'Sullivan JB, Mahan CM, Charles D, Dandrow RV. Screening criteria for high-risk gestational diabetic patients. $A m \mathrm{~J}$ Obstet Gynecol 1973; 116: 895-900.

12. Landy HJ, Gomez-Marin O, O'Sullivan MJ. Diagnosing gestational diabetes mellitus: use of a glucose screen without administering the glucose tolerance test. Obstet Gynecol 1996; 87: 395-400.

13. Schmidt MI, Matos MC, Reichelt AJ, Forti AC, de Lima L, Duncan BB. Prevalence of gestational diabetes mellitus - do the new WHO criteria make a difference? Brazilian Gestational Diabetes Study Group. Diabet Med 2000; 17: 376380.

14. Mello G, Parretti E, Cioni R, Lucchetti R, Carignani L, Martini $\mathrm{E}$, et al. The 75-gram glucose load in pregnancy: relation between glucose levels and anthropometric characteristics of infants born to women with normal glucose metabolism. Diabetes Care 2003; 26: 1206-1210. 